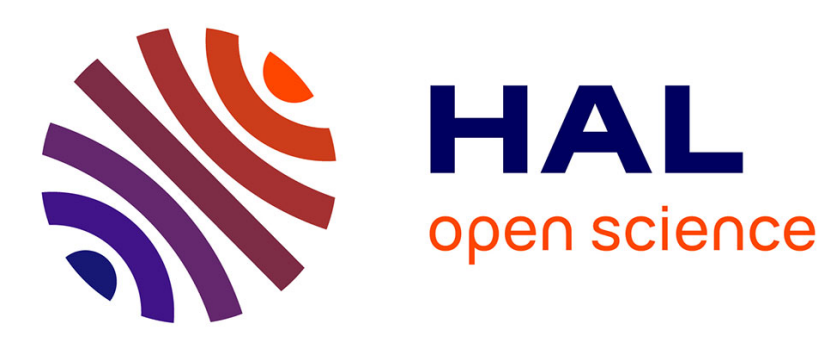

\title{
Numerical method for the Compton scattering operator.
}

Christophe Buet, Stéphane Cordier

\section{To cite this version:}

Christophe Buet, Stéphane Cordier. Numerical method for the Compton scattering operator.. Series on Advances in Mathematics for Applied Sciences, 2003, Lectures notes on discretization of the Boltzmann equation, pp.63. hal-00076898

\section{HAL Id: hal-00076898 \\ https://hal.science/hal-00076898}

Submitted on 3 Sep 2006

HAL is a multi-disciplinary open access archive for the deposit and dissemination of scientific research documents, whether they are published or not. The documents may come from teaching and research institutions in France or abroad, or from public or private research centers.
L'archive ouverte pluridisciplinaire HAL, est destinée au dépôt et à la diffusion de documents scientifiques de niveau recherche, publiés ou non, émanant des établissements d'enseignement et de recherche français ou étrangers, des laboratoires publics ou privés. 


\title{
Numerical method for the Compton scattering operator.
}

\author{
C. Buet ${ }^{1}$, S. Cordier ${ }^{2}$ \\ ${ }^{1}$ Commissariat à l'Énergie Atomique, 91680 Bruyères-le-Châtel \\ email christophe.buet@cea.fr \\ ${ }^{2}$ Laboratoire MAPMO, UMR 6628, \\ Université Orléans, 45067 Orléans, France \\ email Stephane.Cordier@univ-orleans.fr
}

\begin{abstract}
In this paper, we present a new discretization of the Kompaneets equation. This equation can be derived from the Quantum Boltzmann equation for cross sections localized around small changes in the energy. The two collision operators share the same properties (conservation, entropy and steady states). The numerical methods are designed in order to preserve these properties which insures the correct long time behaviour. A supplementary difficulty arises for initial data with a density larger than a critical one, associated with a Planck distribution. In this case, the equilibrium state are the sum of a smooth Planck distribution plus a Delta measure on zero energy. The scheme is able to deal with this singularity.
\end{abstract}

\section{Introduction}

We are interested in the dynamics of a low energy, homogeneous and isotropic photon gas that interacts via Compton scattering with a low energy electron gas in thermodynamical equilibrium.

In this paper, we first review the main properties of the Quantum Boltzmann equation (QBE) and its "grazing" collision limit that is called the Kompaneets equation (which is a Fokker-Planck like equation). We will recall recent result of Escobedo and Mischler [EM, EM2] about the equilibrium state of such equation that behaves under some conditions as a "concentration near the origin" (section $2)$. 
In section 3.1, we present a numerical scheme for the QBE that preserves the properties of the equation described above.

Then, we present a numerical scheme for the Kompaneets equations that has the same properties i.e. are compatible with all the properties of the continuous equation. This allow us to observe the "concentration" phenomena. We present two ways for deriving this scheme. The first way is based on the asymptotic that transform the QBE into the Kompaneets equation at the discrete level. The second one is based on the same ideas as the Chang-Cooper paper [CC] . These two methods lead surprisingly to the same scheme up to a multiplicative constant (that goes to 1 when the mesh is refined) and this fact allows us to use either method usually devoted to Boltzmann like equation (e.g. entropy decay) or to Fokker-Planck type equation (Maximum principle).

We shall also deal with the difficulty due to the mass concentration phenomena. For this, we shall defined generalized Bose-Einstein distribution function (with negative value of $\nu$ ) that converge (when the mesh is refined) toward the Planck distribution plus a Dirac measure at origin.

We then illustrate the schemes on the following result by Escobedo et al. [E3] : even if the initial density is smaller than the Planck one, the solution of the Kompaneets equation (with the boundary conditions) is not always global in time (on the contrary, the solutions of the QBE are well defined for any time) provided that the initial density (arbitrary small) is close enough to the origin. This is due to a balance between the "Burgers" term (with a negative velocity that push the particle toward the origin and the diffusion term that spreads the solution. We shall observe this "blow-up" in finite time.

This paper is related to previous works of the authors on the Boltzmann or Fokker-Planck-Landau operator [BCDL, BC] . In these papers, numerical discretizations are designed in order to be compatible with relevant physical properties such as conservation laws and entropy decay using Discrete Velocities Method. These methods allow to insure the correct large time behaviour i.e. the trend to thermodynamical equilibrium. The numerical efficiency of the proposed algorithms have been tested also for the non homogeneous case (where the distribution function depend on the space variable too) in a paper by the authors and F. Filbet $[\mathrm{BCF}]$.

\section{Quantum Boltzmann and Kompaneets Equa- tion}

In this section, we briefly present the equation we are interested in, namely the Quantum Boltzmann equation and its "grazing" limit the so called Kom- 
paneets equation. We refer to $[\mathrm{K}]$ for the original paper on these equations and to [EM, EM2] for a more recent and mathematical presentation.

\subsection{The Quantum Boltzmann Equation}

We consider an isotropic and homogeneous photon gas scattered by cold electrons at thermodynamical equilibrium. The distribution function of the photons $f$ depends on time and on the energy variable $k: f(k, t)$ represents the number of photons that have energy $k \geq 0$ at time $t \geq 0$. This function $f$ obeys to the following scaled quantum Boltzmann equation (QBE)

$$
k^{2} \frac{\partial f}{\partial t}=\int_{0}^{\infty} b\left(k^{\prime}, k\right)\left(f^{\prime}(1+f) \exp (-k)-f\left(1+f^{\prime}\right) \exp \left(-k^{\prime}\right)\right) d k^{\prime},
$$

where we omit the variable $k$ and $t$ for simplicity and $f^{\prime}$ as usual denotes $f\left(k^{\prime}, t\right)$. The so called cross section $b$ - positive and symmetric- is related to the probability for a given particle at energy level $k$ to be scattered to the energy level $k^{\prime}$. The exponential terms represents the distribution function of electrons. Defining $g=$ $k^{2} f$, equation (2.1) can be rewritten as

$$
\frac{\partial g}{\partial t}=\int_{0}^{\infty} b\left(k^{\prime}, k\right)\left(g^{\prime}\left(k^{2}+g\right) \exp (-k)-g\left(k^{\prime 2}+g^{\prime}\right) \exp \left(-k^{\prime}\right)\right) d k^{\prime},
$$

Let us define the function $h$ of two variable as

$$
h(g, k) \stackrel{\text { def }}{=} \frac{g}{k^{2}+g} .
$$

In the reminder, we shall also note $h=h(g, k)=h(g(k), k)$. Using this notation, the $\mathrm{QBE}$ equation (2.2) reads in the more symmetric form

$$
\frac{\partial g}{\partial t}=\int_{0}^{\infty} b\left(k^{\prime}, k\right) e^{k} e^{k^{\prime}}\left(k^{2}+g\right)\left(k^{\prime 2}+g^{\prime}\right)\left(h\left(g^{\prime}, k^{\prime}\right) e^{k^{\prime}}-h(g, k) e^{k}\right) d k^{\prime},
$$

In the reminder of the paper, we write $Q B$ for the Quantum Boltzmann operator defined above as the right hand side in (2.4).

Multiplying (2.4) by a test function $\Psi(k)$, integrating over $k$ and replacing $k$ by $k^{\prime}$, we have the weak symmetrized formulation of the QB operator

$$
\begin{aligned}
\int_{0}^{\infty} \frac{\partial g}{\partial t} \Psi(k) d k= & \frac{1}{2} \int_{0}^{\infty} \int_{0}^{\infty}\left(\Psi(k)-\Psi\left(k^{\prime}\right)\right) b\left(k^{\prime}, k\right) e^{-k} e^{-k^{\prime}} \\
& \left(k^{2}+g\right)\left(k^{\prime 2}+g^{\prime}\right)\left(h\left(g^{\prime}, k^{\prime}\right) e^{k^{\prime}}-h(g, k) e^{k}\right) d k^{\prime} d k .
\end{aligned}
$$


Using this weak formulation, one obtains easily the unique law of conservation for the QBE, that is the conservation of the total density

$$
N \stackrel{\text { def }}{=} N(g)=\int_{0}^{\infty} g d k, \quad \frac{d}{d t} N(g)=0 .
$$

The second main property of the QBE is the decay of entropy. We first define the function $s(x, k)$ of two variables as

$$
s(x, k) \stackrel{\text { def }}{=} x \log (x)+k^{2} \log \left(k^{2}\right)+k x-\left(k^{2}+x\right) \log \left(k^{2}+x\right),
$$

and thefunctional entropy, $H$ as

$$
H(g) \stackrel{\text { def }}{=} \int_{0}^{\infty} s(g(k), k) d k
$$

Then, $H$ decays in time

$$
\frac{d}{d t} H \leq 0
$$

This property, so called $H$-theorem can be easily checked, at least formally, on the weak formulation of the QBE. Indeed, we have

$$
\frac{d}{d t} s(g(k), k)=\log \left(\frac{g \exp (k)}{k^{2}+g}\right) \frac{d}{d t} g(k) .
$$

Hence, the result is obtained by choosing

$$
\Psi(k)=\log (h(g, k) \exp (k))=\log \left(\frac{g \exp (k)}{k^{2}+g}\right) .
$$

in the weak form (2.5) using that $b \geq 0$.

\subsection{The Kompaneets equation}

When the cross section $b$ concentrates on small modification of the energy (this asymptotic can be related to the grazing collision limit of the classical Boltzmann equation, see $[\mathrm{DV}]$ ), one obtains a Fokker-Planck type equation that has first been derived by Kompaneets in $[\mathrm{K}]$. This equation shares the same properties (total density, $N$, conservation and entropy decay) with the QBE. Let us refer to [EM2] for recent mathematical work on this equation. It is proved that solution of the QBE tends to the solution of the Kompaneets equation which we shall now describe.

In this "grazing" collision limit, which is detailled in the above reference and presented at a discrete level in section 3.2.1, the QBE becomes

$$
\partial_{t} f(k, t)=k^{-2} \partial_{k}\left(k^{4}\left(f+f^{2}+\partial_{k} f\right)\right),
$$


i.e. the sum of a convective term, a Burgers like nonlinear term and a diffusive term with weights $k^{2}$ and $k^{4}$. This so called Kompaneets equation (denoted by $\mathrm{K}$ equation in this article) can be equivalently written as

$$
\partial_{t} f(k, t)=k^{-2} \partial_{k}\left(k^{4}(1+f)^{2}\left(\frac{f}{1+f}+\partial_{k} \frac{f}{1+f}\right)\right) .
$$

A third expression of the Kompaneets equation reads

$$
\partial_{t} g=\partial_{k}\left(\exp -(k+\mu)\left(g+k^{2}\right)^{2} \partial_{k} \frac{h(g, k)}{h\left(B_{\mu}, k\right)}\right),
$$

where we use $g=k^{2} f, h$ defined by (2.3) and the so called Bose-Einstein state (see section 2.3)

$$
B_{\mu}(k) \stackrel{\text { def }}{=} \frac{k^{2}}{\exp (k+\mu)-1},
$$

for $\mu \geq 0$. This is easily checked using the identity $h\left(B_{\mu}(k), k\right)=\exp -(k+\mu)$.

The properties of this equation can be verified on its weak formulation. Multiplying (2.10) by a test function $\Psi(k)$ and integrating over $k$, we have the weak formulation of the Kompaneets equation

$$
\int_{0}^{\infty} \frac{\partial g}{\partial t} \Psi(k) d k=\int_{0}^{\infty} \partial_{k} \Psi(k) \exp -(k+\mu)\left(g+k^{2}\right)^{2} \partial_{k} \frac{h(g, k)}{h\left(B_{\mu}, k\right)} d k .
$$

The $H$ functional entropy (resp. $N$ total density) defined by (2.7) (resp. by (2.6)) is decaying (resp. constant) as it can be checked using $\Psi=\log (h(g, k) \exp (k))$ (resp. $\Psi=1$ ) in the above weak form.

As usual (see [BC, BCF, BCDL]), the formulations are (formally) equivalent at the continuous level. This is no more the case once discretized : the properties of the discretized equation will depend of the form which is discretized.

\subsection{Entropy and equilibrium states}

Let us now turn to the equilibrium states or stationary solutions of QB or $\mathrm{K}$ equations. Such functions should minimize the entropy $H$ for a fixed total density $N$. The function $s$ being concave with respect to its first variable, it is easy to find the minimum as zero of its derivative (with respect to $x$ ) for any fixed $k$. This gives the so-called Bose-Einstein distribution $B_{\mu}(k)$, defined by $(2.11)$, with $\mu$. The coefficient $\mu$ has to be positive in order that the density is finite i.e. the function $B_{\mu}$ is integrable. The limit case $\mu=0$ is related to the Planck distribution. This parameter $\mu$ is a function of the density - we denote by $N_{\mu}=N\left(B_{\mu}\right)$ - 
and this function is decreasing since the Bose-Einstein states are ordered : if $\mu<\nu$ then $B_{\mu}>B_{\nu}$. The application $\mu \mapsto N_{\mu}$ maps $[0, \infty)$ into $\left.] 0, N\left(B_{0}\right)\right]$.

Thus, there is a critical or maximal density $N_{0}=N\left(B_{0}\right)$ that corresponds to the Planck distribution $\nu=0$. For any initial density with a density greater than $N_{0}$, there is no classical equilibrium state. Caflisch and Levermore prove in [CD] that, in this case, the minimum of entropy is realized by the Planck distribution $B_{0}$ plus a Dirac measure located at $k=0, \delta_{0}$, that does not change the value of the entropy $H$ i.e. $H\left(B_{0}+\alpha \delta_{0}\right)=H\left(B_{0}\right)$.

We summarize these results in the following $\mathrm{H}$-theorem for the QB or the $\mathrm{K}$ equation:

Theorem 2.1 For any positive weak solution of $Q B$ or $K$ equation with initial data $g^{0}$, one has $\frac{d}{d t} H(g) \leq 0$ with equality if and only if $g=M$, the equilibrium state $M$ being defined by

- if $N\left(g^{0}\right) \leq N_{0}$ then $M=B_{\mu}$ with $\mu \geq 0$ such that $N\left(g^{0}\right)=N\left(B_{\mu}\right)$

- if $N\left(g^{0}\right)>N_{0}$ then $M=B_{0}+\left(N\left(g^{0}\right)-N_{0}\right) \delta_{0}$

For the details and the proof of this result we refer to [CD, EM, EM2] .

Escobedo and Mischler study the evolution problem and prove the convergence in large time of the (weak) solution of QBE toward these equilibrium states starting from smooth initial data. More precisely, they prove that, in the second case i.e. for an initial density larger than the critical one, the regular part of the solution converges to the Planck distribution (in $L^{1}(\varepsilon, \infty)$ for any $\varepsilon>0$ ) and the reminding part of the density condensates near the origin.

Let us precise the result for the Cauchy problem associated with the QB or the $\mathrm{K}$ equation with an initial data $g^{0} \in S$ defined by

$$
S=\left\{g \in L_{[0, \infty[}^{1} \cap L_{[0, \infty[}^{\infty}, k g \in L_{[0, \infty[}^{1} g \log (g) \in L_{[0, \infty[}^{1}\right\} .
$$

The existence and uniqueness for the QBE in this space is proved in [EM, EM2] . However, this hypothesis for $g^{0}$ is not sufficient to guarantee the existence of a global positive solution for the $\mathrm{K}$ equation. Blow up in finite time can occur even if $N\left(g^{0}\right) \leq N(P)$. A sufficient condition to ensure existence of global in time, positive solution is that $g^{0} \leq P$. 
We also use another form of the entropy, so called relative entropy. We note $H(g \mid M)=H(g)-H(M)$ with $M$ the equilibrium state associated with $g^{0} \in S$, defined in the theorem 2.1. A simple calculation gives

$$
H(g \mid M)=\int\left(g \log (g / M)-\left(k^{2}+g\right) \log \left(\left(k^{2}+g\right) /\left(k^{2}+M\right)\right)\right) d k .
$$

\subsection{A Maximum principle and a Czizar-Kullback like in- equality}

We first prove a maximum principle for positive and global solutions $g \in$ $C^{1}([0, \infty[, S)$ for the $\mathrm{QB}$ or the $\mathrm{K}$ equation. Our result can be stated as

Lemma 2.1 Let $g \in C^{1}([0, \infty[, S)$ such that the $H$-theorem holds i.e. the functional defined by (2.7) decays

- if there exists $\mu$ such that $\alpha \stackrel{\text { def }}{=} \sup \frac{h\left(g^{0}, k\right)}{h\left(B_{\mu}, k\right)}<+\infty$ then $\sup \frac{h(g, k)}{h\left(B_{\mu}, k\right)} \leq \alpha$.

- if there exists $\mu$ such that $\frac{h\left(g^{0}, k\right)}{h\left(B_{\mu}, k\right)} \geq 1$ then $\frac{h(g, k)}{h\left(B_{\mu}, k\right)} \geq 1$.

Using that for any $\mu \geq 0, h\left(B_{\mu}, k\right)=\exp -(k+\mu)$, we can write the QBE $(2.5)$ as

$$
\begin{aligned}
\int_{0}^{\infty} \frac{\partial g}{\partial t} \Psi(k) d k= & \frac{1}{2} \int_{0}^{\infty} \int_{0}^{\infty}\left(\Psi(k)-\Psi\left(k^{\prime}\right)\right) b\left(k^{\prime}, k\right) \exp -(k+\mu) \exp -\left(k^{\prime}+\mu\right) \\
& \left(k^{2}+g\right)\left(k^{\prime 2}+g^{\prime}\right)\left(\frac{h\left(g^{\prime}, k^{\prime}\right)}{h\left(B_{\mu}, k^{\prime}\right)}-\frac{h(g, k)}{h\left(B_{\mu}, k\right)}\right) d k^{\prime} d k
\end{aligned}
$$

and the $\mathrm{K}$ equation as (2.12) These weak formulations are useful to prove the decay of the relative entropy. The proof of this Lemma is postponed in Appendix A.

Note that in the first case $\left(\sup \frac{h\left(g^{0}, k\right)}{h\left(B_{\mu}, k\right)}<+\infty\right)$ the solution behaves as $g \sim C k^{2} \exp (-k)$ for sufficiently large $k$.

Remark 2.2 This result gives an upper bound of the solution only for $k>$ $\max (0, \log (\alpha))$.

For some special cases we deduce the following corollary

Corollary 2.3 If $g \in C^{1}\left(\left[0, \infty[, S)\right.\right.$ and if there exist $\mu_{1}$ and $\mu_{2}$ such that $B_{\mu_{2}} \leq$ $g^{0} \leq B_{\mu_{1}}$ then $B_{\mu_{2}} \leq g \leq B_{\mu_{1}}$. 
Proof. We have just to prove the second inequality since the first is exactly the second part of Lemma 2.1. Since the function $h(g, k)$ is monotone in $g$ for fixed $k$, we have

$$
\frac{h\left(g^{0}, k\right)}{h\left(B_{\mu_{1}}, k\right)}=\alpha \leq 1
$$

Lemma 2.1 gives $h(g, k) \leq h\left(B_{\mu_{1}}, k\right)$ and using again the fact that $h(g, k)$ is increasing in the variable $g$ the result follows.

Concerning the trend to equilibrium, we prove the following Czizar-Kullback like inequality

Lemma 2.2 If $g \in C^{1}\left(\left[0, \infty[, S)\right.\right.$ and $\sup \frac{h\left(g^{0}, k\right)}{h\left(B_{0}, k\right)}<+\infty$ then for any $k_{0}>0$ there exists a constant $C$ depending only on $k_{0}, N\left(g^{0}\right)$ and $\sup \frac{h\left(g^{0}, k\right)}{h\left(B_{0}, k\right)}$ such that

$$
\|g-M\|_{L_{\left[k_{0}, \infty[\right.}^{1}} \leq C H(g \mid M)^{\frac{1}{2}}
$$

Proof. The proof is divided in two parts, one part for "small" $k$ and the second one for "large" $k$. The entropy $H(g \mid M)$ is defined by (2.13). Using the identities $1-h(g, k)=\frac{k^{2}}{g+k^{2}}$ and $\frac{1-h(M, k)}{1-h(g, k)}=\frac{k^{2}+g}{k^{2}+M}$, we can rewrite $H$ as

$$
H(g \mid M)=\int\left(k^{2}+g\right)\left(h(g, k) \log \frac{h(g, k)}{h(M, k)}+(1-h(g, k)) \log \frac{1-h(g, k)}{1-h(M, k)}\right) d k .
$$

Then, using the inequality, see [kullback,]

$$
p_{1} \log \left(\frac{p_{1}}{p_{2}}\right)+\left(1-p_{1}\right) \log \left(\frac{1-p_{1}}{1-p_{2}}\right) \geq 2\left(p_{1}-p_{2}\right)^{2}+\frac{3}{4}\left(p_{1}-p_{2}\right)^{4} \geq 2\left(p_{1}-p_{2}\right)^{2},
$$

which holds for any $0 \leq p_{1} \leq 1$ and $0 \leq p_{2} \leq 1$, for $p_{1}=h(g, k)$ and $p_{2}=h(M, k)$, we obtain

$$
H(g \mid M) \geq 2 \int\left(k^{2}+g\right)\left(\frac{g}{k^{2}+g}-\frac{M}{k^{2}+M}\right)^{2} d k .
$$

Note that

$$
\left(k^{2}+g\right)\left(\frac{g}{k^{2}+g}-\frac{M}{k^{2}+M}\right)^{2}=\frac{k^{4}|g-M|^{2}}{\left(k^{2}+g\right)\left(k^{2}+M\right)^{2}} .
$$

Then, for any $k_{0}>0$ and $R>k_{0}$, we obtain using Cauchy-Schwarz inequality

$$
\int_{k_{0}}^{R}|g-M| d k \leq\left(\int_{k_{0}}^{R} \frac{\left(k^{2}+g\right)\left(k^{2}+M\right)^{2}}{k^{4}} d k\right)^{1 / 2}\left(\int_{k_{0}}^{R} \frac{k^{4}|g-M|^{2}}{\left(k^{2}+g\right)\left(k^{2}+M\right)^{2}} d k\right)^{1 / 2} .
$$


It is easy to check that there exists $C_{1}>0$ such that

$$
\left(\int_{k_{0}}^{R} \frac{\left(k^{2}+g\right)\left(k^{2}+M\right)^{2}}{k^{4}} d k\right)^{1 / 2} \leq C_{1}\left(k_{0}, R, N\left(g_{0}\right)\right)
$$

with $C_{1}\left(k_{0}, R\right)$ which goes to $+\infty$ as $k_{0} \rightarrow 0$ or $R \rightarrow \infty$. Thus, we have

$$
\int_{k_{0}}^{R}|g-M| d k \leq C_{1}\left(k_{0}, R, N\left(g_{0}\right)\right) H(g \mid m)
$$

We now consider the case of "large" $k$. Define $y(t)$ as

$$
y(t)=H\left(M^{t}\right), \quad M^{t} \stackrel{\text { def }}{=} M+t(g-M) .
$$

Thus

$$
y^{\prime}(t)=\int_{k \geq 0}(g-M) \partial_{g} s\left(M^{t}\right) d k
$$

and using $M^{t=0}=M$, which is the minimum of $s$, we have $y^{\prime}(t=0)=0$,

$$
y^{\prime \prime}(t)=\int_{k \geq 0}(g-M)^{2} \partial_{g g} s\left(M^{t}\right) d k=\int_{k \geq 0} \frac{k^{2}(g-M)^{2}}{M^{t}\left(M^{t}+k^{2}\right)} d k .
$$

Using now the Taylor's formula with integral reminder gives us

$$
y(t)=H(M)+\frac{1}{2} \int_{0}^{1}(1-z) \int_{k \geq 0} \frac{k^{2}(g-M)^{2}}{M^{z}\left(M^{z}+k^{2}\right)} d k d z
$$

Using $M^{t}=M+t(g-M) \leq M+g$, we obtain

$$
y(t) \geq H(M)+\frac{1}{2} \int_{k \geq 0} \frac{k^{2}(g-M)^{2}}{(M+g)\left(M+g+k^{2}\right)} d k
$$

that is, by taking $t=1$ i.e. $M^{t=1}=g$ and $y(t=1)=H(g)$

$$
\int_{k \geq 0} \frac{k^{2}(g-M)^{2}}{(M+g)\left(M+g+k^{2}\right)} d k \leq 2 H(g \mid M) .
$$

Using again Cauchy-Schwarz inequality, for all $R \geq 0$

$$
\int_{k \geq R}|g-M| d k \leq\left(\int_{k \geq R} \frac{(M+g)\left(k^{2}+M+g\right)}{k^{2}} d k\right)^{1 / 2}\left(\int_{k \geq R} \frac{k^{2}|g-M|^{2}}{(M+g)\left(g+k^{2}+M\right)} d k\right)^{1 / 2},
$$

and

$$
\int_{k \geq R} \frac{(M+g)\left(k^{2}+M+g\right)}{k^{2}} d k=\int_{k \geq R}(g+M) d k+\int_{k \geq R} \frac{(g+M)^{2}}{k^{2}}
$$


the first term of the r.h.s. is bounded by $2 N\left(g^{0}\right)$. For the second term, since we have assumed that $\alpha=\sup \frac{h\left(g^{0}, k\right)}{h\left(B_{0}, k\right)}<+\infty$, and using lemma 2.1, we have $\sup \frac{h(g, k)}{h\left(B_{0}, k\right)}<\alpha$ and, for $k>\max (\log (\alpha), 0)$, we have $g \leq \frac{\alpha k^{2} \exp (-k)}{1-\alpha \exp (-k)}$. Choosing $R>\log (\alpha)$ there exists a constant $C_{2}=C_{2}\left(N\left(g^{0}\right), R\right)$ such that

$$
\int_{k \geq R}|g-M| d k \leq C_{2}\left(\int_{k \geq R} \frac{k^{2}|g-M|^{2}}{(M+g)\left(g+k^{2}+M\right)^{2}} d k\right)^{1 / 2},
$$

which gives us

$$
\int_{k \geq R}|g-M| d k \leq C_{2} H(g \mid M)^{1 / 2} .
$$

Using $R=\alpha$ in (2.16) and in (2.17), we have

$$
\|g-M\|_{L_{\left[k_{0}, \infty[\right.}^{1}} \leq C H(g \mid M)^{1 / 2},
$$

with $C=C\left(k_{0}, N\left(g^{0}\right), \sup \frac{h\left(g^{0}, k\right)}{h(P, k)}\right)=\max \left(C_{1}, C_{2}\right)$ as we claimed.

Remark 2.4 It can be checked that the constant $C=C\left(k_{0}, N\left(g^{0}\right), \sup \frac{h\left(g^{0}, k\right)}{h(P, k)}\right)$ blows up as $k_{0}$ tends to 0 . However if there exists $\mu$ such that $g^{0} \leq B_{\mu}$ and $\mu>0$, then by means of the corollary 2.3, $g \leq B_{\mu}$. This implies that $\frac{g+M}{k^{2}}$ is bounded by $\frac{1}{\exp (k+\mu)-1}$ which is $L^{1}$ and this is still true with $k_{0}=0$ and thus the constant only depends on the initial data $C=C\left(g^{0}\right)$.

In some case, the entropy permits to control the trend of $g$ toward $M$.

Lemma 2.3 For initial data such that $g^{0}<B_{0}$, the solution of the $K$ equation verifies $\lim _{t \rightarrow+\infty} H(g \mid M)=0$

Proof. We know that for such initial data that there exists a global in time positive solution, [EM,EM2] . Thus, $H(g \mid M)$ is well defined at any time, $C^{1}$ in $t$, monotone decreasing and positive. Thus, there exists $a \geq 0$ such that $\lim _{t \rightarrow+\infty} H(g \mid M)=a$ and an increasing sequence $t_{n} \rightarrow \infty$ such that $H^{\prime}\left(g_{n} \mid M\right)\left(t_{n}\right) \rightarrow 0$, with $g_{n}=g\left(t_{n}\right)$, as $n \rightarrow \infty$.

Since for all $n, g_{n} \leq B_{0}$, using Dunford-Pettis theorem, up to an extraction $g_{n} \rightarrow \bar{g}$, in the sense of measures and $\bar{g}$ is such that $H^{\prime}(\bar{g} \mid M)=0$, that is $\bar{g}=M$. We can also prove that, up to an extraction, $\left\{g_{n}\right\}$ is bounded in $W_{\left[k_{0}, \infty[\right.}^{1,1}$, for any $k_{0}>0$. The $\mathrm{K}$ equation can be written as

$$
\frac{\partial g}{\partial t}=\frac{\partial F}{\partial k}
$$


with $F$ defined by $F=\left(k^{2}-2 k\right) g+g^{2}+k^{2} \frac{\partial g}{\partial k}$. Thus

$$
\frac{\partial g}{\partial k}=\frac{1}{k^{2}}\left(F-\left(k^{2}-2 k\right) g-g^{2}\right)
$$

and then

$$
\int_{k_{0}}^{+\infty}\left|\frac{\partial g}{\partial k}\right| d k \leq \int_{k_{0}}^{+\infty} \frac{1}{k^{2}}|F| d k+\int_{k_{0}}^{+\infty} \frac{1}{k^{2}}\left|\left(k^{2}-2 k\right) g+g^{2}\right| d k .
$$

Since $g \leq B_{0}$, the second term of the r.h.s. of the above inequality (2.18) is clearly bounded by a constant which does not depend on $k_{0}$. Using CauchySchwarz inequality, we have

$$
\int_{k_{0}}^{+\infty} \frac{1}{k^{2}}|F| d k \leq\left(\int_{k_{0}}^{+\infty} \frac{1}{k^{2}} \frac{g\left(k^{2}+g\right)}{k^{4}} d k\right)^{1 / 2}\left(\int_{k_{0}}^{+\infty} \frac{1}{k^{2}} \frac{F^{2}}{g\left(k^{2}+g\right)} d k\right)^{1 / 2} .
$$

Let us now consider the production of entropy

$$
\frac{d H}{d t}=\int_{k} \log \left(\frac{h(g)}{h\left(B_{0}\right)}\right) \frac{d g}{d t} d k .
$$

Then, using $\frac{\partial g}{\partial t}=\frac{\partial F}{\partial k}$ and integrating by parts, one get

$$
\frac{d H}{d t}=\int_{k} \partial_{k}\left(\log \left(\frac{h(g)}{h\left(B_{0}\right)}\right)\right) F d k
$$

or, using the definition (2.3) of $h$,

$$
\frac{d H}{d t}=-\int_{0}^{+\infty} \frac{1}{k^{2}} \frac{F^{2}}{g\left(k^{2}+g\right)} d k
$$

and then

$$
\int_{k_{0}}^{+\infty} \frac{1}{k^{2}}|F| d k \leq\left(\int_{k_{0}}^{+\infty} \frac{g\left(k^{2}+g\right)}{k^{4}} d k\right)^{1 / 2}\left\|\frac{d H}{d t}\right\|^{1 / 2} .
$$

Using one more time $g \leq B_{0}$, one can also verify that

$$
\int_{k_{0}}^{+\infty} \frac{1}{k^{2}} \frac{g\left(k^{2}+g\right)}{k^{4}} d k
$$

is bounded by a constant which depends only of $k_{0}$. The result can be summarized as,for each $k_{0}$ there exist two constants $C_{1}$ and $C_{2}\left(k_{0}\right)$ such that

$$
\int_{k_{0}}^{+\infty}\left|\frac{\partial g}{\partial k}\right| d k \leq C_{1}+C_{2}\left(k_{0}\right)\left\|\frac{d H}{d t}\right\|^{1 / 2} .
$$


By construction, the sequence $g_{n}$ is such that $H^{\prime}(g)$ tends to zero. Thus, the sequence $H^{\prime}\left(g_{n}\right)$ is bounded and thus for any $k_{0}>0$, the sequence $g_{n}$ is bounded in $W_{\left[k_{0}, \infty[\right.}^{1,1}$.

Now by means of this estimate, Helly theorem and diagonal extraction, up to an extraction we have $g_{n} \rightarrow M$ a.e.. One can also easily check that if $0 \leq g \leq$ $B_{0}$ then $|s(g, k)| \leq\left|s\left(B_{0}, k\right)\right|$ and $s\left(B_{0}, k\right)$ is indeed in $L^{1}([0, \infty[)$. Thus, using Lebesgue dominated convergence theorem

$$
\lim _{t_{n} \rightarrow+\infty} H\left(g_{n}\right)=H(M)
$$

that is $a=0$.

\section{Semidiscretization}

Let us now turn to the discretization in the energy variable of the QB and of the $\mathrm{K}$ equation. We consider an uniform grid in $k$ denoted by

$$
k_{i}=i h
$$

for $i=0 \cdots n$, with $k_{0}=n h$.

\subsection{Semidiscretization for the QBE}

We shall detail the scheme for two asymptotic cross sections, the uniform one and the grazing or concentrated one.

Let us recall the QBE, once restricted to a bounded domain

$$
\frac{\partial g}{\partial t}=\int_{0}^{k_{0}} b\left(k, k^{\prime}\right)\left(\left(k^{2}+g\right) e^{-k} g^{\prime}-\left(k^{\prime 2}+g^{\prime}\right) e^{-k^{\prime}} g\right) d k^{\prime}
$$

and the associated symmetrized weak formulation reads

$$
\int_{0}^{k_{0}} \Psi \frac{\partial g}{\partial t}=\frac{1}{2} \int_{0}^{k_{0}} \int_{0}^{k_{0}} b\left(\left(k^{2}+g\right) e^{-k} g^{\prime}-\left(k^{\prime 2}+g^{\prime}\right) e^{-k^{\prime}} g\right)\left(\Psi-\Psi^{\prime}\right) d k^{\prime} d k,
$$

for any test function $\Psi$. The discretization of (3.1) is based on a standard quadrature formula for the above integral

$$
\frac{\partial g_{i}}{\partial t}=h \sum_{j=0}^{N} b\left(k_{i}, k_{j}\right)\left(g_{j}\left(k_{i}^{2}+g_{i}\right) e^{-k_{i}}-g_{i}\left(k_{j}^{2}+g_{j}\right) e^{-k_{j}}\right) .
$$


This gives a numerical method that is conservative and entropy decaying. Indeed, from (3.3) we have the discrete analogue of (3.2)

$$
\sum_{i=0}^{N} \Psi_{i} \frac{\partial g_{i}}{\partial t}=\frac{h^{2}}{2} \sum_{i=0}^{N} \sum_{j=0}^{N} b\left(k_{i}, k_{j}\right) g_{i} g_{j}\left(\frac{\left(k_{i}^{2}+g_{i}\right) e^{-k_{i}}}{g_{i}}-\frac{\left(k_{j}^{2}+g_{j}\right) e^{-k_{j}}}{g_{j}}\right)\left(\Psi_{i}-\Psi_{j}\right) .
$$

Then, using the above weak discretized formulation, we obtain the discrete version of the $\mathrm{H}$-theorem for the QBE with the discrete entropy defined by $H(g)=h \sum_{i=0}^{N} s\left(g_{i}, k_{i}\right)$, that is $\frac{d H}{d t} \leq 0$. By construction Bose-Einstein functions are equilibrium points of the discrete $\mathrm{QBE}$. The relations defining the discrete equilibrium states, $\frac{d H}{d t}=0$, imply that (with $\left.P_{i}=\left(B_{0}\right)_{i}\right)$

- $\frac{h\left(g_{i}, k_{i}\right)}{h\left(P_{i}, k_{i}\right)}=\frac{h\left(g_{j}, k_{j}\right)}{h\left(P_{j}, k_{j}\right)}$ for all $i, j>0$.

- $g_{0}\left(g_{j}-\exp \left(-k_{j}\right)\left(k_{j}^{2}+g_{j}\right)\right)=0$, for all $j$.

If $g_{0}(t=0)=0$, one can check on (3.3) that $g_{0}(t)=0$ for all $t$ and, in this case, the equilibrium states $M$ associated to $g$ are Bose-Einstein function $B_{\mu}$ eventually with $\mu$ negative. This fact happens, as we will see also for the $\mathrm{K}$ equation, in the case of $N\left(g^{0}\right)>N\left(B_{0}\right)$.

If $g_{0}(0) \neq 0$, the situation is quite different: if $N\left(g^{0}\right) \leq N\left(B_{0}\right)$ then necessarily $M=B_{\mu}$ with a positive $\mu$, which implies that $\lim _{t \rightarrow+\infty} g(k=0, t)=0$, and, if $N\left(g^{0}\right)>N\left(B_{0}\right)$, then $M=B_{0}+\alpha \delta_{0}$ with $\alpha=N\left(g^{0}\right)-N\left(B_{0}\right)$ (this is not proved because $M_{0}=0$ and $M=B_{\mu}$ with negative $\mu$ is also a possible equilibrium state for the differential system).

These nonlinear ordinary differential systems can be very simplified in the two particular cases of uniform and concentrated cross sections.

\subsubsection{Uniform cross section}

When $b\left(k, k^{\prime}\right)=\bar{b}(k) \bar{b}\left(k^{\prime}\right)$, the evaluation of the double integral (3.2) reduces to two (simple) moments :

$$
\frac{\partial g}{\partial t}=\left(\int_{0}^{k_{0}} \bar{b}\left(k^{\prime}\right) g^{\prime} d k^{\prime}\right) \bar{b}(k)\left(k^{2}+g\right) e^{-k}-\left(\int_{0}^{k_{0}} \bar{b}\left(k^{\prime}\right)\left(k^{\prime 2}+g^{\prime}\right) e^{-k^{\prime}} d k^{\prime}\right) \bar{b}(k) g .
$$

Note that this expression is compatible with the measure values solution of the form $g=g_{\text {reg }}+\alpha \delta_{0}$ considered in [EM2] (which satisfied a system of equation for both $g_{r e g}(k, t)$ and $\alpha(t)$, described in [EM] ). One replaces the integral by a 
discrete sum. Assume $\bar{b}(k)=1$ for example (as in [EM2] ). Let us note $M_{0}$ the discrete density and define the first moment as

$$
M_{1}=h \sum_{i=1}^{n}\left(k_{i}^{2}+g_{i}\right) e^{-k_{i}} .
$$

We consider the following explicit scheme

$$
\frac{g_{i}^{n+1}-g_{i}^{n}}{\Delta t_{n}}=M_{0} k_{i}^{2} e^{-k_{i}}+\left(M_{0} e^{-k_{i}}-M_{1}^{n}\right) g_{i}^{n} .
$$

The conservation of density can be verified by summing the r.h.s. in the above equations. Positivity is preserved at any iteration, provided that

$$
\Delta t_{n} \leq \frac{1}{M_{1}^{n}}
$$

Indeed, multiplying (3.6) by $e^{k_{i}}$ and summing over $i$ gives

$$
\begin{aligned}
\frac{M_{1}^{n+1}-M_{1}^{n}}{\Delta t_{n}} & =M_{0}\left(\sum k_{i}^{2} g_{i}^{n} e^{-2 k_{i}}+e^{-2 k_{i}}\right) h-M_{1}^{n} h \sum e^{k_{i}} g_{i}^{n} \\
& \leq M_{0} M_{1}^{n}-M_{1}^{n}\left(M_{1}^{n}-\sum k_{i}^{2} e^{k_{i}} h\right) \\
& \leq C M_{1}^{n}-\left(M_{1}^{n}\right)^{2},
\end{aligned}
$$

with $C=M_{0}+\sum k_{i}^{2} e^{k_{i}} h$. Then,

$$
M_{1}^{n+1} \leq M_{1}^{n}+C \Delta t_{n} M_{1}^{n}-\Delta t_{n}\left(M_{1}^{n}\right)^{2},
$$

and using $\Delta t_{n}=\frac{1}{M_{1}^{n}}$, we have by induction that $M_{1}^{n} \leq C$ and the time steps are bounded from below.

\subsection{Semidiscretization for the Kompaneets equation}

For the discretization of the $\mathrm{K}$ equation we proceed in two ways. The first one is based on the scheme for the QBE and the fact that for concentrated cross section around $k=k^{\prime}$, the $\mathrm{K}$ equation is the asymptotic limit of the QBE, see [EM] . The second one is adapted from the method proposed by Chang and Cooper, see $[\mathrm{CC}]$, for linear equation of Fokker-Planck type.

\subsubsection{Concentrated cross section}

One consider a cross section sequences on the form

$$
b^{\varepsilon}\left(k, k^{\prime}\right)=\frac{1}{\varepsilon^{3}} \tilde{b}\left(\frac{k-k^{\prime}}{\varepsilon}\right),
$$


where $\tilde{b}$ is a positive and even function. This gives the K equation (when $\tilde{b}\left(k, k^{\prime}\right)=$ $\left.e^{k / 2} e^{k^{\prime} / 2}\right)$.

If we assume, for simplicity, that $\tilde{b}$ is compactly supported with $\operatorname{supp}(\tilde{b})=$ ] $-3 / 2,3 / 2[$ and choose $\varepsilon=h$. The cut-off/smoothing parameter $\varepsilon$ is equal to the mesh size. Then, in the double sum (3.4), only the terms such that $(i-j)= \pm 1$ do not vanish. We obtain the following scheme

$$
\begin{aligned}
\frac{\partial g_{i}}{\partial t}= & \frac{\tilde{b}(1)}{h^{2}} \sum_{i=0}^{n} g_{i+1}\left(k_{i}^{2}+g_{i}\right) e^{h / 2}-g_{i}\left(k_{i+1}^{2}+g_{i+1}\right) e^{-h / 2}+ \\
& +g_{i-1}\left(k_{i}^{2}+g_{i}\right) e^{-h / 2}-g_{i}\left(k_{i-1}^{2}+g_{i-1}\right) e^{h / 2}
\end{aligned}
$$

Note that this system (with $\tilde{b}(1)=1$ ) can be also written as

$$
\begin{aligned}
\frac{\partial g_{i}}{\partial t}= & \frac{1}{h^{2}}\left(g_{i+1} k_{i}^{2} e^{h / 2}+g_{i-1} k_{i}^{2} e^{-h / 2}-\left(k_{i+1}^{2} e^{-h / 2}+k_{i-1}^{2} e^{h / 2}\right) g_{i}\right)+ \\
& +\frac{1}{h^{2}}\left(e^{h / 2}-e^{-h / 2}\right)\left(g_{i} g_{i+1}-g_{i} g_{i-1}\right) .
\end{aligned}
$$

The first part corresponds to a tridiagonal linear system and the second part to the non linear Burgers term $f^{2}$. One can write this system in the form

$$
\frac{\partial g_{i}}{\partial t}=\frac{1}{h}\left(F_{i+\frac{1}{2}}-F_{i-\frac{1}{2}}\right)
$$

with $F_{-1 / 2}=F_{N+1 / 2}=0$ and for $i=0, \ldots, n-1$

$$
\begin{aligned}
F_{i+\frac{1}{2}} & =\frac{1}{h}\left(\exp \left(\frac{h}{2}\right) k_{i}^{2} g_{i+1}-\exp \left(-\frac{h}{2}\right) k_{i+1}^{2} g_{i}\right)+\left(\exp \left(\frac{h}{2}\right)-\exp \left(-\frac{h}{2}\right)\right) g_{i} g_{i+1} \\
& =\frac{1}{h} \exp \left(-\frac{k_{i}+k_{i+1}}{2}\right)\left(g_{i}+k_{i}^{2}\right)\left(g_{i+1}+k_{i+1}^{2}\right)\left(\frac{h\left(g_{i+1}, k_{i+1}\right)}{h\left(B_{i+1}, k_{i+1}\right)}-\frac{h\left(g_{i}, k_{i}\right)}{h\left(B_{i}, k_{i}\right)}\right) \\
& =\frac{1}{h} h\left(B_{\mu}\left(\frac{k_{i}+k_{i+1}}{2}\right), \frac{k_{i}+k_{i+1}}{2}\right)\left(\frac{h\left(g_{i+1}, k_{i+1}\right)}{h\left(B_{i+1}, k_{i+1}\right)}-\frac{h\left(g_{i}, k_{i}\right)}{h\left(B_{i}, k_{i}\right)}\right),
\end{aligned}
$$

for any bose -Einstein state $B_{\mu}$, since $h\left(B_{\mu}, k\right)=\exp (k+\mu)$. This is the consistency of this scheme for the Kompaneets equation. Indeed, the flux of the $\mathrm{K}$ equation $(2.10)$ is

$$
\mathcal{F}(g, k)=\left(g+k^{2}\right)^{2} \exp (-k) \frac{\partial}{\partial k}\left(\frac{h(g, k)}{h(P, k)}\right) .
$$

Using a taylor expansion around $k_{i+\frac{1}{2}}=\frac{k_{i}+k_{i+1}}{2}$ we obtain

$$
F_{i+\frac{1}{2}}=\mathcal{F}\left(g_{i+\frac{1}{2}}, k_{i+\frac{1}{2}}\right)+\mathrm{O}\left(h^{2}\right)
$$




\subsubsection{Chang and Cooper method}

We now follow the method proposed by Chang and Cooper $[\mathrm{CC}]$. We use from (2.9) which is close to the linear Fokker-Planck expression and discretize the fluxes at the interface $F_{i+\frac{1}{2}}$ between $k_{i}$ and $k_{i+1}$ in a standard centered finite difference for the diffusion part

$$
\left(\partial_{k} \frac{f}{1+f}\right)_{i+\frac{1}{2}}=\frac{1}{h}\left(\frac{f_{i+1}}{1+f_{i+1}}-\frac{f_{i}}{1+f_{i}}\right)=\frac{f_{i+1}-f_{i}}{h\left(1+f_{i+1}\right)\left(1+f_{i}\right)},
$$

a linear combination for the convective part :

$$
\left(\frac{f}{1+f}\right)_{i+\frac{1}{2}}=\left(1-\delta_{i+\frac{1}{2}}\right) \frac{f_{i+1}}{1+f_{i+1}}-\delta_{i+\frac{1}{2}} \frac{f_{i}}{1+f_{i}},
$$

where $0 \leq \delta_{i+\frac{1}{2}} \leq 1$ has to be define further, and, using an harmonic average for the "diffusion coefficients"

$$
\left(k^{4}(1+f)^{2}\right)_{i+\frac{1}{2}}=k_{i}^{2} k_{i+1}^{2}\left(1+f_{i}\right)\left(1+f_{i+1}\right) .
$$

This last choice permits to simplify the denominator in the diffusion discretization which is now linear in $f$. Then, the flux can be written after some simple calculus as (with $g_{i}=k_{i}^{2} f_{i}$ )

$$
F_{i+\frac{1}{2}}=\delta_{i+\frac{1}{2}} g_{i}\left(g_{i+1}+k_{i+1}^{2}\right)+\left(1-\delta_{i+\frac{1}{2}}\right) g_{i+1}\left(g_{i}+k_{i}^{2}\right)+\frac{1}{h}\left(k_{i}^{2} g_{i+1}-k_{i+1}^{2} g_{i}\right) .
$$

The coefficients $\delta_{i+\frac{1}{2}}$ are now to be determined. We shall choose them in order that the Bose-Einstein equilibrium states are preserved by this scheme. We impose that for $g_{i}=M_{i}=\frac{k_{i}^{2}}{e^{k_{i}+\alpha}-1}$ with any real value of $\alpha$, the flux vanishes. This gives

$$
\delta_{i+\frac{1}{2}}=\frac{1}{h}-\frac{1}{e^{h}-1} .
$$

Note that this coefficients are independent of $i$ for such a uniform grid and have the following Taylor expansion for small $h$ :

$$
\delta_{i+\frac{1}{2}}=\frac{1}{2}-\frac{1}{12} h+\frac{1}{720} h^{3}-\frac{1}{30240} h^{5}+O\left(h^{6}\right) .
$$

This means that when $h \rightarrow 0$, the scheme becomes a symmetrized approximation of the convective part.

Using this choice (3.11), the formulae (3.10) can be simplified into

$$
F_{i+\frac{1}{2}}=g_{i+1}\left(g_{i}+k_{i}^{2}\right)+\frac{1}{e^{h}-1}\left(k_{i}^{2} g_{i+1}-k_{i+1}^{2} g_{i}\right) .
$$


The same expression can be found for the flux $F_{i-\frac{1}{2}}$

$$
F_{i-\frac{1}{2}}=g_{i}\left(g_{i-1}+k_{i-1}^{2}\right)+\frac{1}{e^{h}-1}\left(k_{i-1}^{2} g_{i}-k_{i}^{2} g_{i-1}\right) .
$$

Then, we obtain the same semidiscretization (using the notation $F_{-1 / 2}=$ $\left.F_{n+1 / 2}=0\right)$ as in (3.9) up to a multiplicative factor that goes to 1 as $h \rightarrow 0$

$$
\frac{e^{h / 2}-e^{-h / 2}}{h}=1-\frac{1}{24} h^{2}+\frac{7}{5760} h^{4}+O\left(h^{5}\right) .
$$

Remark 3.1 Note that, for a non uniform grid, the same method applies, replacing $h$ by its local value $\Delta k_{i+\frac{1}{2}}=\left(k_{i+1}-k_{i}\right)$. However, the two schemes are no more proportional although the ratio converges to 1 (when the mesh is refined i.e. $\max _{i} \Delta k_{i+\frac{1}{2}} \rightarrow 0$ ).

\subsubsection{Properties of the two schemes}

A discrete integration by parts gives the weak form for the two above schemes:

$$
\sum_{i=0}^{n} \Psi_{i} \frac{\partial g_{i}}{\partial t}=-\frac{1}{2} \sum_{i=0}^{n-1}\left(\Psi_{i+1}-\Psi_{i}\right) F_{i+\frac{1}{2}}
$$

Thus, if we define the discrete density $N(g)$ as $\sum_{i=0}^{n} h g_{i}$ and the discrete entropy as $H(g)=\sum_{i=0}^{n} h s\left(g_{i}, k_{i}\right)$ one verifies the conservation of density $\left(\frac{d}{d t} N(g)=0\right)$ and the decay of entropy.

Indeed we have

$$
\frac{d}{d t} H(g)=\sum_{i=0}^{N} h \partial_{g} s\left(g_{i}, k_{i}\right) \frac{d g_{i}}{d t}=\sum_{i=0}^{N} h \log \left(\frac{h\left(g_{i}, k_{i}\right)}{h\left(P_{i}, k_{i}\right)}\right) \frac{d g_{i}}{d t},
$$

thus using (3.10)

$$
\frac{d}{d t} H(g)=-\sum_{i=0}^{N} h C\left(k_{i}^{2}+g_{i}\right)\left(k_{i+1}^{2}+g_{i+1}\right)\left(\log \left(\lambda_{i+1}\right)-\log \left(\lambda_{i}\right)\right)\left(\lambda_{i+1}-\lambda_{i}\right),
$$

with $\lambda_{i+1}=\frac{h\left(g_{i+1}, k_{i+1}\right)}{h\left(P_{i+1}, k_{i+1}\right)}, C=\exp \left(-\frac{k_{i}+k_{i+1}}{2}\right)$ for the flux (3.10) or $C=\frac{\exp \left(-\left(k_{i}+\mu\right)\right)}{\exp (h)-1}$ for the flux (3.12), and $C$ is strictly positive. Using the classical inequality $(x-$ y) $(\log (x)-\log (y)) \geq 0$, we have

$$
\frac{d}{d t} H(g) \leq 0
$$




\subsubsection{Existence of global positive solution.}

We write system (3.8) by factorizing the gain term $G(g)$ and the loss term $L(g)$ as usual for Boltzmann equation

$$
\frac{d g_{i}}{d t}=\frac{1}{h^{2}}\left(G(g)_{i}-L(g)_{i} g_{i}\right)
$$

with $G(g)_{i} \geq 0$ and $L(g)_{i} \geq 0$. We have the existence local in time using the Cauchy-Lipschitz theorem starting from $g_{i}^{0}>0$ for all $i>0$. Then, the loss term being bounded (for a given initial density $N$ ), we have that the solution remains positive. Finally, using the conservation of density, we have an upper bound for the semidiscretized solution $g_{i} \leq N / h$ and the solution is global in time. Note that this upper bound does not prevent concentration.

\subsubsection{Discrete equilibrium state}

We shall prove that the discrete equilibrium states are the generalized BoseEinstein distribution functions. We restrict ourselves to the case $f^{0}(0)<\infty$ that is $g^{0}(0)=0$. It is easy to verify that if $g_{0}(t=0)=0$ then $g_{0}(t)=0$. Thus, the distribution function is discretized on $\left[h, k_{0}\right]$ i.e. for $i=1 \cdots n$. From $(3.14)$, it is easy to verify that $\frac{d}{d t} H(g)=0$ if and only if $g=B_{\mu}$ with $\mu$ eventually negative.

Let us consider, for any fixed density $N$, the discrete Bose-Einstein distribution

$$
B_{\alpha}^{h}(k)=\frac{k^{2}}{e^{k+\alpha}-1} \chi_{k \geq h},
$$

where $\alpha$ is such that

$$
N^{h} \stackrel{\text { def }}{=} \sum_{i=1}^{n} B_{\alpha}^{h}\left(k_{i}\right) h=N .
$$

Note that $N_{h}$ is decreasing with $\alpha$ and is one to one from $]-h, \infty[$ to $[0, \infty[$. Thus, any arbitrary density $N$ can be associated with a generalized discrete Bose-Einstein state provided that the mesh is refined enough such that $N>N^{0}$ or $N h<k_{0}$. When $n \rightarrow \infty$ or $h \rightarrow 0$, one has for $N \leq N^{0}$

$$
N_{h} \rightarrow N\left(B_{\mu}\right)
$$

and for $N>N^{0}, N_{h} \rightarrow B_{0}+\left(N-N^{0}\right) \delta_{0}$. Indeed, in the second case, one has $-h \leq \alpha \leq 0$ and thus $\alpha \rightarrow 0$ as $h \rightarrow 0$. One can check easily that if $N_{h}\left(g^{0}\right)>N_{h}\left(B_{0}\right)$, the equilibrium state associated to $g^{0}$ is a Bose-Einstein state $B_{\alpha}^{h}$ with $\alpha \in[-h, 0[$. Let us now precise the relation between such equilibrium state and $B_{0}+\left(N\left(g^{0}\right)-N\left(B_{0}\right)\right) \delta_{0}$. For simplicity, we consider the problem in continuous variable $k$, thus $N_{h}$ is now

$$
N_{h} \stackrel{\text { def }}{=} \int_{k \geq h} M_{h}(k) d k \text {. }
$$


We shall prove the following result

Proposition 3.2 For any $N>0, B_{\alpha}^{h}$ converge when $h \rightarrow 0$ in the sense of distribution toward the continuous equilibrium functions that is, when $N \leq N_{0}$, there exists $\beta>0$ such that $B_{\alpha}^{h} \rightarrow B_{\beta}$ i.e. $\alpha \rightarrow \beta$ as $h \rightarrow 0$ and when $N>N^{0}$

$$
B_{\alpha}^{h} \rightarrow B_{0}+\left(N-N^{0}\right) \delta_{0}
$$

where $B_{0}$ is the Planck distribution and $N^{0}$ its density. Moreover, the associated entropy converges i.e. $H\left(M_{h}\right) \rightarrow H\left(B_{0}\right)$ when $N>N^{0}$ and $H\left(B_{\alpha}^{h}\right) \rightarrow H\left(B_{\beta}\right)$ when $N \leq N^{0}$.

The proof is postponed in Appendix B and can be applied for a finite domain $\left[0, k_{0}\right]$ or with discrete measure (sums instead of integrals).

\subsubsection{Maximum principle for the discrete equation Kompaneets}

Let us prove a stronger result that the existence of a positive solution when the initial data is in between two Bose-Einstein functions. Indeed, as for the linear Fokker-Planck operator, the Kompaneets equation satisfies a Maximum principle that is verified on its discretized version.

Proposition 3.3 Let $g$ a solution of (3.8). If there exists $\mu$ such that $g(t=$ $0, k) \leq B_{\mu}(k)$ (resp. $\left.g(t=0, k) \geq B_{\mu}(k)\right)$, then, for all $t>0$, one has $g(t, k) \leq$ $B_{\mu}(k)$ (resp. $\left.g(t, k) \geq B_{\mu}(k)\right)$.

Proof. First, one checks easily that the scheme (3.8) can be written in the form

$$
\frac{d g_{i}}{d t}=C_{i+\frac{1}{2}} D\left(\frac{h(g, k)}{h(B, k)}\right)_{i+\frac{1}{2}}-C_{i-\frac{1}{2}} D\left(\frac{h(g, k)}{h(B, k)}\right)_{i-\frac{1}{2}}
$$

where we note $h(g, k)=\frac{g(k)}{k^{2}+g(k)}$ and $D$ is a finite difference operator defined as

$$
D \phi_{i-\frac{1}{2}}=\phi_{i+1}-\phi_{i}
$$

and $C_{i+\frac{1}{2}}$ are non negative coefficients (with the notation $C_{-1 / 2}=C_{N+1 / 2}=0$ ) that depend on the function $g$ and of $k$.

Note that for any Bose-Einstein function $B_{\mu}$,

$$
h\left(B_{0}, k\right)=\frac{B_{0}}{k^{2}+B_{0}}=\exp (-k)=h\left(B_{\mu}, k\right) \exp (-\mu)
$$


Therefore, one can change $B_{0}$ into any Bose-Einstein $B_{\mu}$ in formula (3.16) with coefficient multiplied by $\exp (-\mu)$. Moreover, $h(g, k)$ is a increasing function of $g$ since

$$
\frac{\partial h(g, k)}{\partial g}=\frac{k^{2}}{\left(k^{2}+g^{2}\right)} .
$$

We denote by

$$
\left.\lambda_{i}=\frac{h\left(g_{i}, k_{i}\right)}{h\left(B_{\mu}\left(k_{i}\right), k_{i}\right.}\right),
$$

then the $\left(\lambda_{i}\right)$ satisfy a system of the same form i.e.

$$
\frac{d \lambda_{i}}{d t}=E_{i+\frac{1}{2}} D(\lambda)_{i+\frac{1}{2}}-E_{i-\frac{1}{2}} D(\lambda)_{i-\frac{1}{2}}
$$

with non negative $E_{i+\frac{1}{2}}$.

Define $\lambda_{S}=\max _{i=1 \cdots N} \lambda_{i}$. The function $\lambda_{S}$ is a piecewise $C^{1}$ function of $t$. By definition of $\lambda_{S}$, we have, $\forall t>0$, there exists a subset $I_{S}(t)$ of $\{1, \cdots, N\}$, such that $\lambda_{i}(t)=\lambda_{S}(t)$ for all $i \in I_{S}(t)$. Thus, $\forall i \in I_{S}(t), \forall j \in\{1, \cdots, N\}, \lambda_{i} \geq \lambda_{j}$ and using the positivity of the coefficients $E$, we obtain that $\frac{d \lambda_{S}}{d t} \leq 0$. The same idea proves that the minimum of $\lambda_{i}$ increases with time.

Remark 3.4 Assume that for some $\mu, g(t=0) \leq B_{\mu}$, then $h(g(t=0), k) \leq$ $h\left(B_{\mu}, k\right)$ or equivalently $\lambda_{S}(0) \leq 1$ then for all $t, \lambda_{S}(t) \leq 1$ i.e. $g(t, k) \leq B_{\mu}(k)$. In the same way, if for some $\mu, g(t=0) \geq B_{\mu}$, then $g(t, k) \geq B_{\mu}(k)$.

Thus, using the Kullback like inequality (2.2), which is also valid for discrete measures, we can prove the following lemma

Lemma 3.1 When $g(t=0) \leq B_{0}$, the distribution function converges toward its equilibrium.

Proof. Using $H(g \mid M)=H(g)-H(M)$, where $M$ is the discrete equilibrium associated to $g$, is decreasing in time and is positive. Thus, there exists a increasing and diverging sequence $t_{k}$ such that $H^{\prime}\left(t_{k}\right) \rightarrow 0$. The zero of the derivative of $H$ are the equilibrium $M$. Hence, $g\left(t_{k}\right)$ goes to $M$ and $H\left(g\left(t_{k}\right) \mid M\right) \rightarrow H(M \mid M)=0$. Since $H(g \mid M)$ is decreasing, we have necessary

$$
\lim _{t \rightarrow \infty} H(g \mid M)=0
$$

and, by lemma 2.2, we obtain, the convergence of $g$ toward its equilibrium $M$.

Remark 3.5 The results of this section are valid for the discrete QBE, the proofs being analogous. 


\section{Time discretization for the Kompaneets equa- tion}

We eliminate explicit time discretization since, when Bose condensation occurs, time step to ensure positivity would be in $\mathrm{O}\left(h^{3}\right)$ to compare with $\Delta t=\mathrm{O}\left(h^{2}\right)$ for classical parabolic problems. Therefore, we shall only consider implicit scheme. We shall see that a fully implicit scheme has good properties but seems hard to implement at a reasonable cost. We propose an alternative implicit scheme with a low cost but for which we cannot prove all the features of the fully implicit scheme. As illustrated by numerical examples, this scheme works well.

\subsection{Fully implicit scheme}

Let us consider an implicit scheme of the form ( $g$ represents $g^{n}$ at iteration $n$ and $\bar{g}$ denotes $g^{n+1}$ )

$$
\bar{g}=g+t Q(\bar{g})
$$

Assume that the scheme is positive i.e. $g^{n}>0 \Rightarrow g^{n+1}>0$, we shall prove that it is automatically entropy decaying i.e. $H^{n+1}=H\left(g^{n+1}\right)<H\left(g^{n}\right)=H^{n}$.

Indeed, $H(g)=\int s(g, k)$ defined by $(2.13)$ and using a second order Taylor expansion, at point $\bar{g}$ with integral reminder, we have

$$
H(\bar{g})-H(g)=\partial_{g} H(\bar{g})(t Q(\bar{g}))+t^{2} \int_{0}^{1}(1-z) Q(\bar{g})^{T} \partial_{g g}^{2} H(\bar{g}+z(g-\bar{g})) Q(\bar{g}) d z
$$

where $\partial_{g} H$ denotes the functional derivative

$$
\partial_{g} H=\int \partial_{g}(s(g, k)) d k=\int \log \left(\frac{g \exp (k)}{k^{2}+g}\right) d k
$$

and we have $\partial_{g} H(\bar{g})(t Q(\bar{g})) \leq 0$. Moreover, the second derivative with respect to $g$ is negative

$$
\partial_{g g}^{2} s(g, k)=\frac{1}{g}-\frac{1}{g+k^{2}} \leq 0,
$$

and this concludes the proof.

The existence of a positive solution for the implicit scheme is ensured by the Brouwer fixed point theorem. We choose $C>0$ such that $C N(g) f+Q(f)$ is a positive operator for all positive $f$ such that the density of $f$ less or equal to $N(g)$. Then (4.17) can be rewritten as ( $f$ denotes $g^{n+1}$ and $g$ denotes $\left.g^{n}\right)$ :

$$
f(1+N(g) C t)=g+N(g) C t\left(f+\frac{Q(f)}{N(g) C}\right) .
$$


The mapping $f \mapsto T(f)$

$$
T(f)=\frac{1}{1+N(g) C t} g+\frac{N(g) C t}{1+N(g) C t}\left(f+\frac{Q(f)}{N(g) C}\right),
$$

is continuous from the convex compact set

$$
E=\{f>0 \text { such that density of } f \text { is less or equal to } N(g)\}
$$

into itself thus the Brouwer fixed point theorem insure the existence of an element $f^{*}$ of $E$ such that $f^{*}=T\left(f^{*}\right)$ and necessarily $f^{*}$ has the same density and energy that $g$.

Despite its good properties, since the implicit scheme is non linear an iterative procedure is needed and have to be stopped before exact convergence.

\subsection{Semi-implicit scheme}

The method we suggest is to treat the linear part implicitly and the non linear part semi-implicitly but in such way that the properties of the semidiscretized system are preserved. As we have seen the differential system (3.8) can be written as

$$
\frac{d}{d t} g_{i}=\frac{1}{h}\left(F(g)_{i+\frac{1}{2}}-F(g)_{i-\frac{1}{2}}\right)
$$

with the fluxes $F(g)_{i+\frac{1}{2}}$ defined by (3.10) and have the structure

$$
F(g)_{i+\frac{1}{2}}=\bar{F} L_{i+\frac{1}{2}}(g)+\bar{F} B_{i+\frac{1}{2}}(g)
$$

with $\bar{F} L_{i+\frac{1}{2}}(g)=a_{i+\frac{1}{2}} g_{i+1}-b_{i+\frac{1}{2}} g_{i}, \overline{F B_{i+\frac{1}{2}}}(g)=c_{i+\frac{1}{2}} g_{i} g_{i+1}$, and $a_{i+\frac{1}{2}}, b_{i+\frac{1}{2}}$ and $c_{i+\frac{1}{2}}$ are non negative, $\overline{F B} B_{i+\frac{1}{2}}(g)$ is the Burgers flux and $\bar{F} L_{i+\frac{1}{2}}(g)$ is the flux for the linear Kompaneets equation.

The semi implicit scheme consists in treating all the fluxes $\bar{F} L_{i+\frac{1}{2}}(g)$ implicitly and to implicit only the term $g_{i+1}$ in the Burgers fluxes $\overline{F B} B_{i+\frac{1}{2}}(g)$. That is, if we assume that $g$ is known at time $t$, we compute $\bar{g}$ at time $t+\vec{\Delta} t$ as:

$$
\bar{g}_{i}=g_{i}+\frac{\Delta t}{h}\left(\bar{F}_{i+\frac{1}{2}}(\bar{g})-\bar{F}_{i-\frac{1}{2}}(\bar{g})+\bar{B}_{i+\frac{1}{2}}(g, \bar{g})-\bar{B}_{i-\frac{1}{2}}(g, \bar{g})\right)
$$

and $\bar{B}_{i+\frac{1}{2}}(g, \bar{g})=c_{i+\frac{1}{2}} g_{i} \bar{g}_{i+1}$. One can verify that the density is preserved i.e. $\sum g_{i}=\sum \bar{g}_{i}$.

This system can be written in the form

$$
(I d-\Delta t M(g)) \bar{g}=g
$$


where $M(g)$ is a tridiagonal matrix and one can check easily that $M(g)$ is also a so-called $L$ matrix, that depends on $g$ i.e. $M(g)_{i i}>0$ and $M(g)_{i j} \leq 0$ for all $i \neq j)$.

The main property of this semi-implicit scheme concerns its positivity:

Lemma 4.1 If $g$ is positive, then $\bar{g}$ defined by (4.21) is positive for all time steps $\Delta t$

Proof. Due to the special structure of $M(g)$, it is easy to check that one can always construct $X>0$ such that $X \in \operatorname{ker} M(g)$ which is equivalent to find $X>0$ such that

$$
\bar{F}_{i+\frac{1}{2}}(X)+B_{i+\frac{1}{2}}(g, X)=0
$$

for all $i$ : start from one index $i_{0}$ by setting $X_{i 0}$ arbitrary strictly positive and in virtue of the positivity of $a_{i+\frac{1}{2}}, b_{i+\frac{1}{2}}, c_{i+\frac{1}{2}}$ relation (4.23) generates positive strictly $X_{i}$.

Then there exists $X>0$ such that $(I d-\Delta t M(g)) X=X$, that is if we set $D$ such that $D_{i, j}=\delta_{i, j} X_{i}$, thus $(I d-\Delta t M) D$ is a diagonal dominant matrix that is $(I d-\Delta t M)$ is a generalized diagonal dominant matrix, which is equivalent to the fact that $M$ is an $M$-matrix or in other words it has a positive inverse positive inverse, [berman-plemons] . This means that the scheme is unconditionally positive (whatever the condensation occurs).

Note that $X$ is related to equilibrium state in the prrof. Concerning the equilibrium states we have also the following result

Lemma 4.2 The scheme (4.21) preserves the equilibrium state.

Proof. If we write $Q(g)$ the operator of the right hand side of (4.20), by construction $Q(g)=0$ if $g$ is an equilibrium state. Since $M(g) g=Q(g)$, (4.22) reads

$$
(I d-\Delta t M(g))(\bar{g}-g)=Q(g)=0 .
$$

Since the matrix $M(g)$ is a M-matrix, then we have $g=\bar{g}$ as we claim it.

One should choose time step of the form $C_{1} h$ where $C_{1}$ is such that the entropy decays. We are not able to exhibit a condition on the time step to ensure the decay of the discrete entropy. But, as we will see on the numerical examples, using a time step corresponding to a the convective equation that is of the form $\Delta t<C \Delta k$ leads to a satisfactory behaviour of $H$ even for singular initial data. 


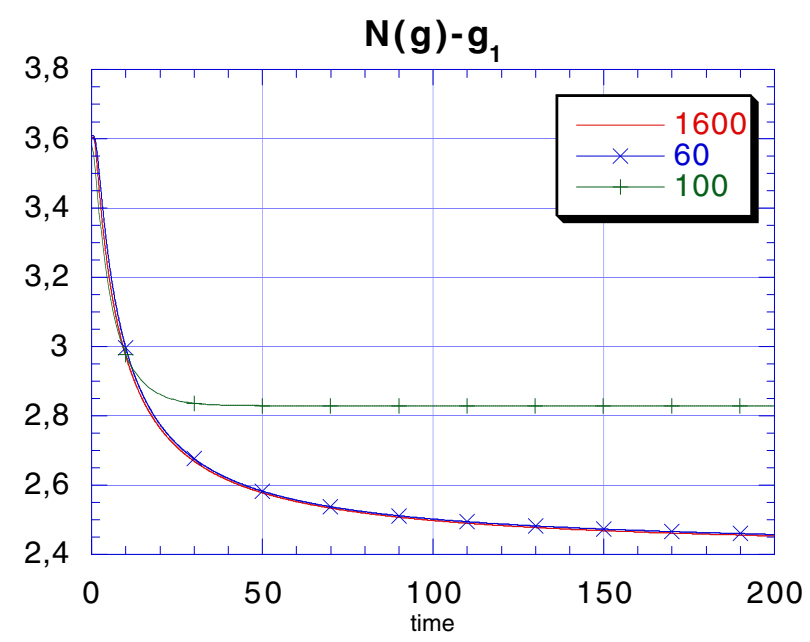

Figure 1: Density in case 1.

\section{$5 \quad$ Numerical tests for the Kompaneets schemes}

We will illustrate the scheme on the two following examples. The first one corresponds to a Planck distribution multiplied by $3 / 2$. In this case, concentration near origin occurs after few iterations since the initial density is greater than the critical one. On the second case, we consider a initial data with a lower density but we still observe a concentration when the initial density is close enough to 0 , as expected after the analysis in $[\mathrm{EHV}]$.

\subsection{Relaxation of $\alpha B_{0}$ with $\alpha=3 / 2$}

We plot the evolution in time of three macroscopic quantities, density for non zero energy, energy and entropy. The runs corresponds to the following three cases

- label "1600" is a reference computation with 1600 points of discretizations and $g_{0}(0)=0$.

- label "100" corresponds to 100 points using the same method and with $g_{0}(0)=0$.

- label "60" denotes the same method but with only 60 points and with an initial data $g_{0}(0) \neq 0$ but very small $\left(10^{-12}\right)$.

More precisely, in Fig1.1 we plot the quantity $\sum_{i \geq 1} g_{i} h$ (the indices start at 1) i.e. the discrete version of $\int_{h}^{k_{0}} g d k$. This quantities is constant when the solution 


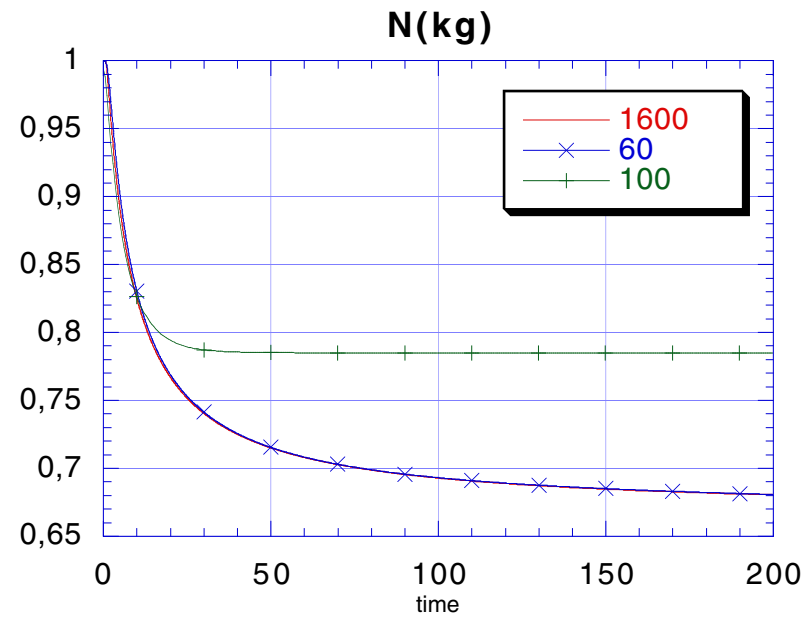

Figure 2: Energy in case 1.

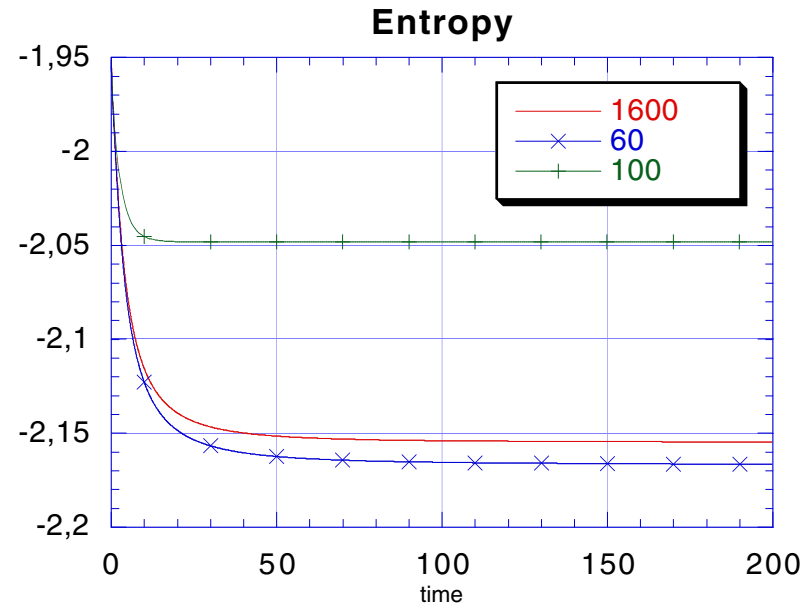

Figure 3: Entropy in case 1. 
remains smooth. It decays when concentration occurs. Fig. 2 (resp. 3) illustrates the evolution of the energy (resp. entropy). The differences of the three runs are in the number of discretization points and in the initial data (at zero energy). Indeed, as explained before (section 3.2.5) there are two ways to discretize the singularity. Either, $g_{0}(0)$ then, $g_{0}(t)=0, \forall t>0$ and the distribution function converges to a generalized Bose-Einstein equilibrium (with $\mu<0$ ) that converges in a distributional sense toward the Planck distribution $\left(B_{0}\right)$ plus a dirac measure at $k=0$ or $g_{0}(0) \neq 0$ and the discretized distribution function converges for $i \geq 0$ toward the Planck distribution, and the remaining density concentrates on $k_{0}=0$.

The presented results show that it is much better to discretize with $g_{0}(0) \neq 0$, in particular in terms of entropy (the entropy is lower with only 60 points and $g_{0}(0) \neq 0$ than with 1600 points and $g_{0}(0)=0$, i.e. generalized Bose-Einstein equilibrium).

\subsection{Initial gaussian distribution.}

In this case, we consider an initial gaussian distribution with a sub-critical density $N<N^{0}$. In the Compton case (or QBE), the solution goes toward its Bose Einstein equilibrium. It has been proved in [EHV] that, for the Kompaneets equation, the solution may not be global in time provided that the initial density is close enough to the origin. Indeed, there is a balance between the Burgers term and the diffusion term : the Burgers part is a convective toward the origin that leads to concentration, whereas the diffusion spreads off the distribution that becomes smoother.

In Fig 1.4, we plot the distribution for different time when the initial data is a Gaussian centred at $k=2$ with 3200 points of discretization. In this case, the diffusion dominates and the distribution function goes to its Bose-Einstein distribution (with $\nu>0$. In Fig 1.5, we plot the same quantities (distribution function versus $k$ at different time) but with an gaussian initial data (same total density, centered at $k=1$ ). In this case, the Burgers term is stronger and the distribution function becomes singular near origin after a finite time $T^{*}$. Clearly, the solution is no more valid for $t>T^{*}$ but the simulation can be continued since the Kompaneets discretization can be interpreted as the discretization of QBE with a small parameter $\varepsilon$ linked to the discretization step $h$ (see section section 3.2.1) for which such a concentration is possible.

On Fig 1.6, we plot the evolution in time of the density for non zero energy and we observe the concentration at time $T^{*}$ : some part of the density goes into the zero energy part of the distribution function. On Fig 1.7 (resp. 1.8), we plot the energy (resp. entropy) versus time. 


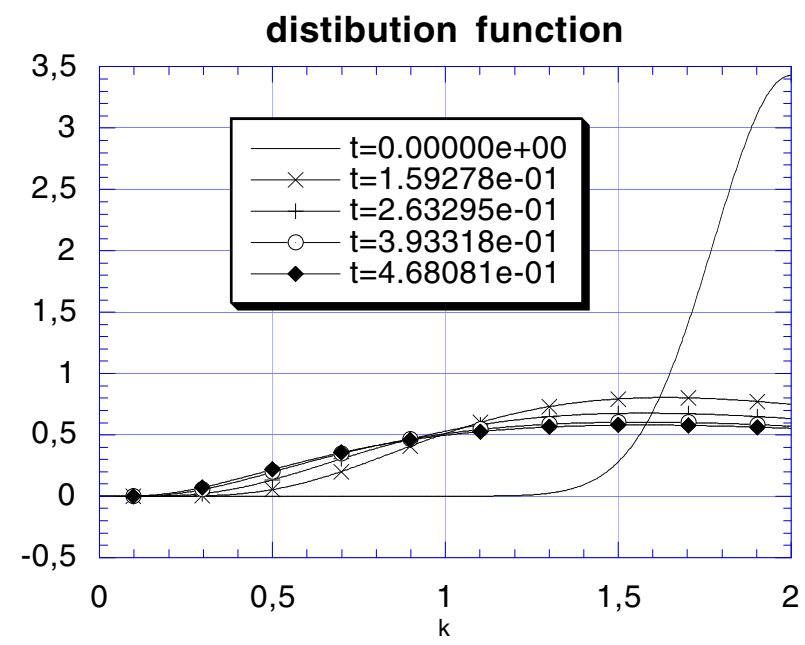

Figure 4: Evolution of the distribution function in case $2, k=2$.

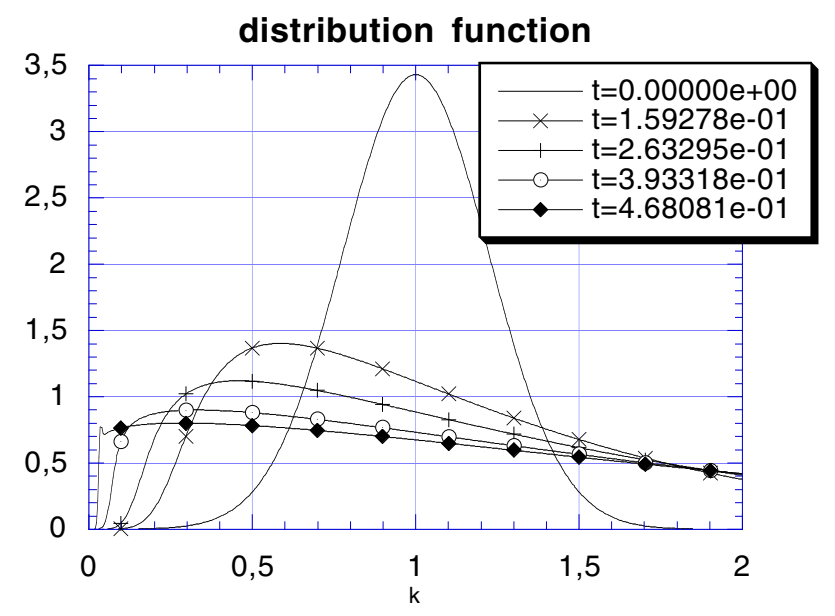

Figure 5: Evolution of the distribution function in case $2, k=1$. 


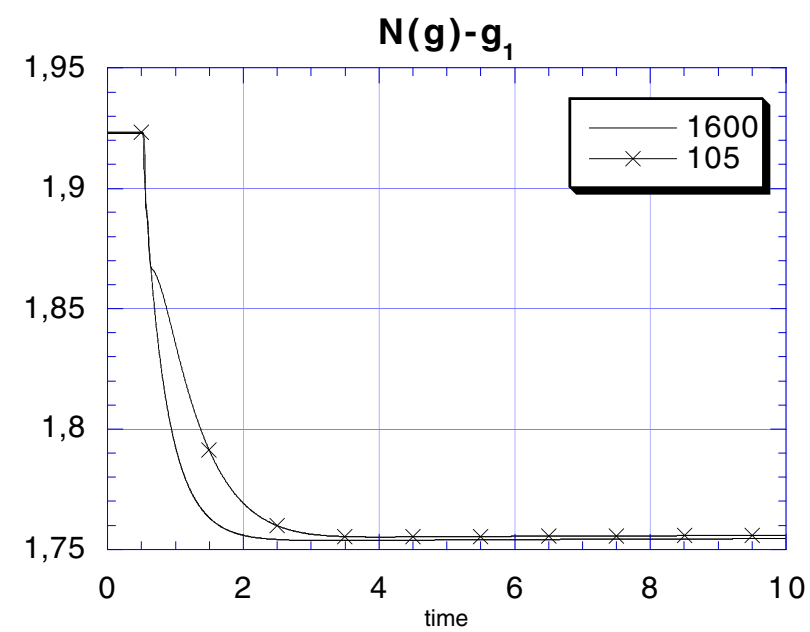

Figure 6: Density versus time in case $2, k=1$.

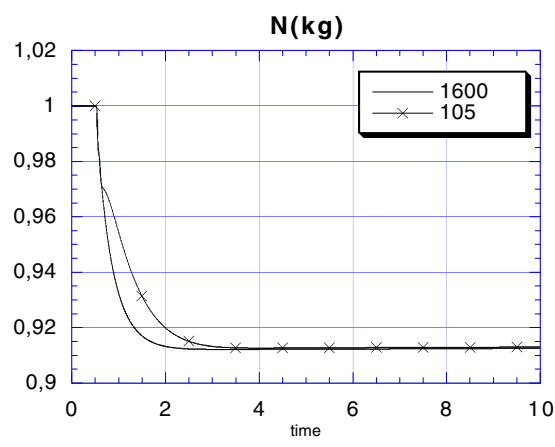

Figure 7: energy

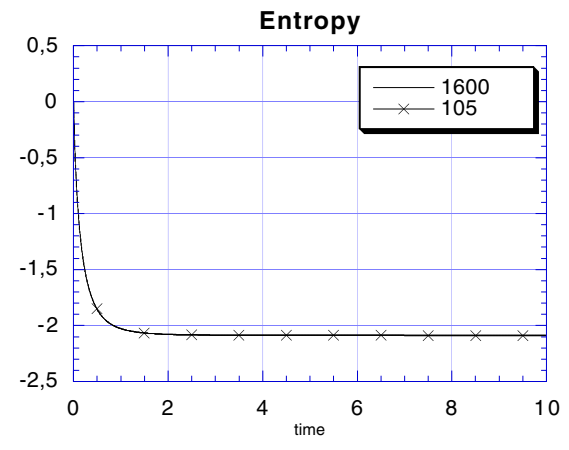

Figure 8: entropy

\section{Conclusions}

The main improvements compared with the previous method investigated in $[\mathrm{P}]$ are

- This method does not require to construct a equilibrium state that usually required an iterative method (which is not always conservative).

- The semi-discretized system preserve all the properties : decay of entropy, conservation of density

- The semi-implicit scheme is unconditionally positive

- Scheme 1 is of order 2 in $k$ 
Moreover, we are able to deal with condensation near the origin when the initial data has a density larger than the Planck one or is to close to the origin, both of these phenomena are illustrated by numerical results. There are two ways to describe this concentration at the discrete level : either $g_{0}(0)=0$ and then, this remains equal 0 at all time - in this case, the equilibrium state are generalized Bose-Einstein state with $\mu<0$ that converge in a distribution sense to the Planck distribution plus a Dirac at $k=0$, see section 3.2.5 and appendix B - or $g_{0}(0) \neq 0$ - in this case, the smooth part converges toward the Planck distribution and the remaining density concentrates on $k=0$. The preliminary numerical results indicate that the second case is better and more precisely, that the first case is unstable : when the initial data at $k=0$ is not equal zero, the scheme converges toward the second representation of the singularity.

Some questions remain to be attend in further works :

- Is the semi-implicit scheme entropic?

- How to construct a full implicit scheme (without iterative method) ?

- Can we insure the same properties for general cross sections with the (QBE).

\section{Appendix A : proof of Lemma 2.2}

Proof. We begin by the first part of the lemma. It is then easy to check that it suffice to prove the result only in the case $\mu=0$, i.e. for $B_{0}$.

For $k \in\left[0, \log ^{+}(\alpha)\right]$ with $\log ^{+}(x)=\max (0, \log (x))$, since the solution is non negative and

$$
h(g, k) \leq 1 \leq \alpha \exp (-k)=\alpha h\left(B_{0}, k\right) .
$$

For $k \in] \log (\alpha), \infty$ [, consider any function $G(x)$ such that $G(x) \geq 0$ for $x \geq 0$, $0<G^{\prime}(x)<\infty$ for $x \geq 0$ and $G(x)=0$ for $x \leq 0$. Then, define $H_{\alpha}(x, k)$ as

$$
H_{\alpha}(x, k)=\int_{x_{0}}^{x} G\left(\frac{h(s, k)}{h\left(B_{0}, k\right)}-\alpha\right) d s
$$

for $k \in] \log ^{+}(\alpha), \infty\left[\right.$ with $x_{0}$ the unique positive solution of $h\left(x_{0}, k\right)=\alpha h\left(B_{0}, k\right)$ (note that $x_{0}=\frac{\alpha k^{2} \exp (-k)}{1-\alpha \exp (-k)}>0$ ) and $H_{\alpha}(x, k)=0$ for $k \leq \log ^{+}(\alpha)$.

Set now

$$
E(t)=\int_{k \geq 0} H_{\alpha}(g(k), k) d k .
$$


It can be checked that by construction $E(t) \geq 0, E(t)$ is continuous and by the hypothesis $E(0)=0$. Moreover, $E(t)$ is $C^{1}$ and we have

$$
\frac{d}{d t} E=\int_{k \geq 0} G\left(\frac{h(g, k)}{h\left(B_{0}, k\right)}-\alpha\right) \frac{d g}{d t} d k
$$

Now using the weakform (2.5) of the QBE, it is easy to see that, since $G\left(\frac{h(g, k)}{h\left(B_{0}, k\right)-\alpha}\right)$ is monotone increasing in the variable $g$ for each $k$. Thus, using the inequality $(x-y)(\phi(x)-\phi(x)) \geq 0$, for any monotone increasing function $\phi, \frac{d}{d t} E \leq 0$.

For the K equation, using the weak form (2.12), it is also clear that $\frac{d}{d t} E \leq 0$. Thus, for both equation, $E(t)=0$ for all time $t$, which is equivalent to $\frac{h(g, k)}{h\left(B_{0}, k\right)} \leq \alpha$ almost everywhere in $k \in] \log ^{+}(\alpha), \infty[$.

For the proof of the second part of the lemma we proceed as for the first part. With the function $G$ defined above, we set $H$ as

$$
H(x, k)=\int_{x_{1}}^{x} G\left(\frac{1-h(s, k}{h\left(B_{0}, k\right)}\right) d s,
$$

if $g \leq x_{1}$. and $H(x, k)=0$ for $g \geq x_{1}$ and define

$$
E(t)=\int_{k \geq 0} H(g, k) d k .
$$

Proceeding as for the first part we obtain that $E(t)$ is monotone decreasing, positive and $E(0)=0$. Thus for any time $E(t)=0$ that is $h(g, k) \geq h\left(B_{0}, k\right)$ a.e. as we have claimed it.

\section{Appendix B : proof of Proposition 3.3}

Proof. As explained above $\alpha$ is a function of $N$ and of $h$.

Define $N(\alpha, h)$ as (for any $h \geq 0$ and $\alpha \geq-h$ )

$$
N(\alpha, h)=\int_{h}^{\infty} \frac{k^{2}}{\exp (k+\alpha)-1} d k .
$$

The equation $N(\alpha, h)=N$ determines implicitly $\alpha$ as a function of $N$ and $h$ If $N>N^{0}$, since $N^{0}=N(0,0), \alpha(N, h) \in[-h, 0[$ thus $\alpha \rightarrow 0$ as $h \rightarrow 0$. Let $\phi$ a test function, we have

$$
L(h)=\int_{0}^{\infty} \phi(k)\left(M_{h}(k)-B_{0}(k)\right) d k=\int_{h}^{\infty} \frac{k^{2} \phi(k)}{\exp (k+\alpha(h))-1} d k-\int_{0}^{\infty} \frac{k^{2} \phi(k)}{\exp (k)-1} d k .
$$


We write $\phi(k)=\phi(0)+k \psi(k)$ where $\psi$ is a $C^{\infty}(0, \infty)$. We know that $\alpha(h) \rightarrow 0$. Thus, we have

$$
\begin{aligned}
L(h)= & \int_{h}^{\infty} k^{2} \phi(0)\left(\frac{1}{\exp (k+\alpha(h))-1}-\frac{1}{\exp (k)-1}\right) d k+ \\
& +\int_{h}^{\infty} k^{3} \psi(k)\left(\frac{1}{\exp (k+\alpha(h))-1}-\frac{1}{\exp (k)-1}\right) d k-\int_{0}^{h} \frac{k^{2} \phi(k)}{\exp (k)-1} d k .
\end{aligned}
$$

The first term gives

$$
\int_{h}^{\infty} k^{2}\left(\frac{1}{\exp (k+\alpha(h))-1}-\frac{1}{\exp (k)-1}\right) d k=N-\int_{h}^{\infty} \frac{k^{2}}{\exp (k)-1} d k \rightarrow N-N^{0} .
$$

- the second can be written as

$$
\left(1-\exp (\alpha(h)) \int_{h}^{\infty} \frac{k^{3} \psi(k) d k}{(\exp (k+\alpha(h))-1)(\exp (k)-1)} d k \rightarrow 0,\right.
$$

and the third term goes to 0 . Thus, we proved that

$$
L(h) \rightarrow\left(N-N^{0}\right) \phi(0) .
$$

This is equivalent to the convergence of $B_{\alpha}^{h}$ toward $B_{0}+\left(N-N_{0}\right) \delta_{0}$ in the distribution sense.

Let us now consider the case $N<N^{0}$. There exists $\beta>0$ such that $N(\beta, 0)=$ $N$. We want to prove that $\alpha(h)$ ( $N$ being fixed) goes to $\beta$ as $h \rightarrow 0$. The function $\alpha$ being decreasing with $h$ and being positive for $h$ small enough, one has $0<\alpha(h)<\beta$. Moreover, $\alpha$ is continuous, then $\lim _{h \rightarrow 0} \alpha(h)=\alpha(0)=\beta$. Then by dominated convergence theorem, we have that $B_{\alpha}^{h}$ tends to $B_{\beta}$ in $L^{1}(0, \infty)$ (and in the distributional sense).

Let us now prove the convergence of the entropy. One has

$$
H(g)=\int_{0}^{\infty}\left(g \log \left(\frac{g}{k^{2}+g}\right)+k^{2} \log \left(\frac{k^{2}}{k^{2}+g}\right)+k g\right) d k .
$$

For the function $B_{\alpha}^{h}$, one easily checks that

$$
\frac{B_{\alpha}^{h}}{k^{2}+B_{\alpha}^{h}}=e^{-(k+\alpha)}, \frac{k^{2}}{k^{2}+B_{\alpha}^{h}}=1-e^{-(k+\alpha)} .
$$

Then, using the expression of $H$ and $B_{\alpha}^{h}$, one obtains

$$
H\left(B_{\alpha}^{h}\right)=-\alpha \int_{0}^{\infty} B_{\alpha}^{h}+\int_{h}^{\infty} k^{2} \log (1-\exp -(k+\alpha)) d k .
$$


The first term is equal to $-\alpha N$ that goes to 0 because $\alpha \rightarrow 0$. Let us note $g_{h}(k)=$ $\log (1-\exp -(k+\alpha))$. We know that $g_{h}$ converges to $g_{0}$ almost everywhere and is integrable on $\left[0, \infty\left[\right.\right.$. Note that $\forall k>\varepsilon$ since $\alpha \leq 0,\left(g_{0}-g_{h}\right)(k) \geq 0$.

$\left(g_{0}-g_{h}\right)(k)=\log \left(\frac{1-\exp -(k)}{1-\exp -(k+\alpha)}\right) \leq \exp (\alpha)(\exp (-\alpha)-1) \int_{h}^{\infty} \frac{k^{2} d k}{\exp -(k+\alpha)-1}$

using $\log (1+h) \leq h$. This proves that

$$
\int_{h}^{\infty}\left(g_{0}-g_{h}\right)(k) d k \rightarrow 0
$$

But,

$$
H\left(B_{\alpha}^{h}\right)-H\left(B_{0}\right)=-\alpha N+\int_{h}^{\infty}\left(g_{h}-g_{0}\right)(k) d k-\int_{0}^{h} g_{0}(k) d k
$$

The last term tends to 0 since $g_{0}$ is integrable. This proves the convergence. In the second case $N<N^{0}$ we know that $\alpha(h)$ is a decreasing function of $h$ that converges toward $\beta>0$ such that

$$
\int_{0}^{\infty} \frac{k^{2} d k}{\exp (k+\beta)-1}=N
$$

The proof given for $N>N^{0}$ can be applied in this case.

\section{Bibliography}

[BP] Berman, A., Plemmons R.J., Nonnegative matrices in the mathematical sciences. Classics in Applied Mathematics, 1994

[B] S. N. Bose, Plancks Gesetz und Lichtquantenhypothese, Z. Phys. 26 (1924), 178-181.

[BCF] Buet, Christophe, Cordier, Stéphane and Filbet, Francis, Comparison of numerical schemes for Fokker-Planck-Landau equation. ESAIM Proc., vol 10, p. 161-181, (2001).

[BC] Buet, C. and Cordier, S., Numerical analysis of conservative and entropy schemes for the Fokker-Planck-Landau equation., SIAM J. Numer. Anal., vol 36, No 3, p. 953-973, (1998).

[BCDL] Buet, C.; Cordier, S.; Degond, P.; Lemou, M. Fast algorithms for numerical, conservative, and entropy approximations of the Fokker-PlanckLandau equation. J. Comput. Phys. 133, No.2, p. 310-322, (1997). 
[CL] R.E. Caflisch, C.D. Levermore, Equilibrium for radiation in a homogeneous plasma, Phys. Fluids 29, 748-752 (1986)

[CC] Chang J.S., Cooper G., A practical difference scheme for FokkerPlanck equations. J. Comput. Phys. 6, 1-16 (1970).

[C] G. Cooper, Compton Fokker-Planck equation for hot plasmas, Phys. Rev. D 3, 2312-2316 (1974)

[DV] L. Desvillettes, C. Villani, On the Spatially Homogeneous Landau Equation for Hard Potentials. Part I: Existence, Uniqueness and Smoothness, CPDE vol. 25, n. 1-2, (2000), pp. 179-259 and Part II: H-Theorem and Applications, CPDE, vol. 25, n. 1-2, (2000), pp. 261-298.

[EHV] M. Escobedo, M.A. Herrero, J.J.L. Velazquez, A nonlinear FokkerPlanck equation modeling the approach to thermal equilibrium in a homogeneous plasma, Trans. Amer. Math. Soc. 350, 3837-3901 (1998)

[EM1] M. Escobedo, S. Mischler, Equation de Boltzmann quantique homogène: existence et comportement asymptotique, Note au C. R. Acad. Sci. Paris 329 Série I, 593-598 (1999)

[EM2] M. Escobedo, S. Mischler: On a quantum Boltzmann equation for a gas of photons, J. Math.Pures Appl. 80, 5 (2001), pp. 471-515.

[EM3] M. Escobedo, S. Mischler, M.A. Valle, On Boltzmann equation for a gas of quantum (and relativistic) particles, work in preparation

[EMV] M. Escobedo, S. Mischler, J.J.L. Velazquez, Specified long time behavior for the Boltzmann-Compton equation, work in preparation

[Ka] O. Kavian, Remarks on the Kompaneets Equation, a Simplified Model of the Fokker Planck equation, to appear in Séminaire J.L. Lions - Collège de France - série rouge (Pitman-Longman-Wesley)

[K] A.S. Kompaneets, The establishment of thermal equilibrium between quanta and electrons, Soviet Physics JETP, (1957)

$[\mathrm{Ku}]$ Kullback, S., On the convergence of discrimination information. IEEE Trans. Information Theory IT-14 1968 765-766

[LLPS] Larsen E.W., Levermore C.D., Pomraning G.C., Sanderson J.G. Discretization methods for one-dimensional Fokker-Planck operators. J. Comput. Phys. 61, 359-390 (1985) 